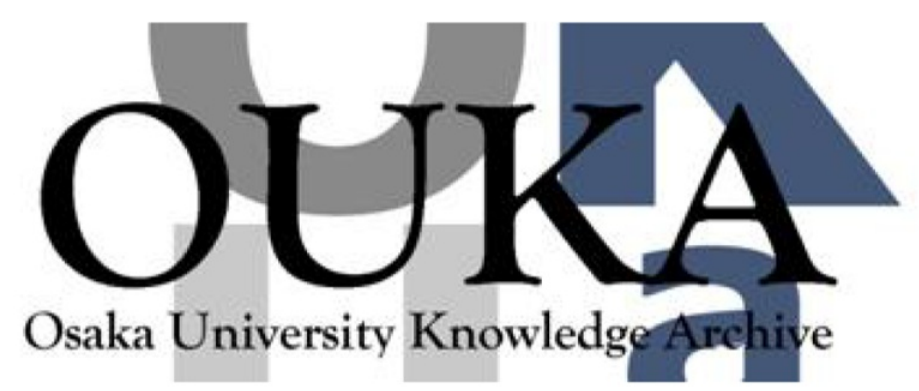

\begin{tabular}{|c|l|}
\hline Title & $\begin{array}{l}\text { Three-dimensional imaging of laser imploded } \\
\text { targets }\end{array}$ \\
\hline Author(s) & $\begin{array}{l}\text { Chen, Yen-Wei; Miyanaga, Noriaki; Yamanaka, } \\
\text { Masanobu et al. }\end{array}$ \\
\hline Citation & Journal of Applied Physics. 68(4) p. 1483-p. 1488 \\
\hline Issue Date & $1990-08-15$ \\
\hline oaire:version & VoR \\
\hline URL & https://hdl. handle. net/11094/3414 \\
\hline rights & \\
\hline Note & \\
\hline
\end{tabular}

Osaka University Knowledge Archive : OUKA

https://ir. Library. osaka-u. ac. jp/

Osaka University 


\section{Three-dimensional imaging of laser imploded targets}

Yen-Wei Chen, Noriaki Miyanaga, Masanobu Yamanaka,

Mitsuo Nakai, Kazuo Tanaka, Katsunobu Nishihara, Tatsuhiko Yamanaka, and Sadao Nakai

Institute of Laser Engineering, Osaka University, Suita, Osaka 565, Japan

(Received 18 December 1989; accepted for publication 19 April 1990)

An $\mathrm{X}$-ray emission computed tomographic technique has been demonstrated to diagnose the laser implosion uniformity of spherical targets. Three-dimensional images of compressed core were reconstructed from five pinhole camera images obtained at different viewing directions by using an iterative algorithm. Good agreement between the experimental results and two-dimensional hydrodynamic simulations showed that the uniformity of initial target shell thickness has a significant influence on implosion symmetry.

\section{INTRODUCTBON}

In laser fusion research, a dense compressed fuel of more than one thousand times liquid density is required to achieve the ignition with a realistic driver energy (a few or several hundred $\mathrm{kJ}$ ). The nonuniformity of laser irradiation, the imperfection of target shell thickness, and resulting hydrodynamic instabilities may seriously inhibit high density compression. The $x$-ray and $\alpha$ particle images obtained by pinhole camera and coded aperture imaging have provided direct evidences of deformation of compressed cores. ${ }^{1,2}$ However, because these images are twodimensional projections of the three-dimensional spherical implosion targets, these may not be enough to evaluate the implosion uniformity.

In order to obtain tomographic pictures of the imploded target, we have applied a computed tomography (CT) technique ${ }^{3}$ developed for medical diagnostics to reconstruct three-dimensional compressed cores from three pinhole camera inages or two uniformly redundant arrays (URA) coded aperture images. ${ }^{4,5}$ In recent experiments, in order to improve the resolution of the reconstruction determined by the amount of tomographic information about the target, we used a CT system with five pinhole cameras to increase the solid angle of the system and incorporated a priori information about the sarget (the reconstruction must be positive and smooth, and that outside the initial target must be zero) to increase the tomographic information. The resolution of the reconstruction has been improved to $30 \mu \mathrm{m}$ from $60 \mu \mathrm{m}$. It becomes possible to study the implosion symmetry by using this technique. In this paper, we present the detailed reconstructions of imploded targets. As a demonstration, both of the implosion targets with and without a uniformity of the initial shell thickness have been reconstructed from five pinhole camera images. The reconstructed compressed cores showed that the uniformity of target shell thickness has a significant influence on the implosion symmetry. The results have been also compared with two-dimensional hydrodynamic computer simulations.

\section{H. RECONSTRUCTION TECHRLUE}

Computed tomography (CT) is one of the inversion techniques to estimate a three-dimensional distribution
$I(x, y, z)$ from its several two-dimensional projections $P_{\theta, \psi}(u, v)$. The coordinate system is shown in Fig. 1. If the self-absorption of $x$ rays in laser-produced plasmas is negligible, the projection $P_{\theta, \psi}(u, v)$ along the viewing direction, $r$, is given by

$$
P_{\phi, \psi}(u, v)=\int I(x, y, z) d r,
$$

where

$$
\left[\begin{array}{l}
u \\
v \\
r
\end{array}\right]=A^{-1}\left[\begin{array}{l}
x \\
y \\
z
\end{array}\right]
$$

and

$$
A=\left(\begin{array}{ccc}
\sin \psi \cos \theta & -\sin \theta & \cos \psi \cos \psi \\
\sin \psi \sin \theta & \cos \theta & \cos \psi \sin \theta \\
-\cos \psi & 0 & \sin \psi
\end{array}\right) .
$$

In order to determine the three-dimensional distribution $I(x, y, z)$ from such two-dimensional projections obtained at different viewing directions, an iterative method known as the algebraic reconstruction technique (ART) ${ }^{6}$ was used. The algorithm is shown as follows;

$$
I^{k+1}\left(x, y_{9} z\right)=I^{k}(x, y, z) \times P_{\theta, \psi}(u, v) / R_{\theta, \psi}^{k}(u, v),
$$

where $f^{k}(x, y, z)$ is the reconstruction obtained after $k$ th iteration, and $R_{\theta, \psi}^{k}(u, v)$ is the projection calculated from $I^{k}$.

As compared to the filtered back-projection method, ${ }^{7}$ which is one of direct (not iterative) methods and usually used in medical CT scanners, the ART method has a great advantage due to the possibility of incorporating constraints in the reconstruction with limited angular projections. As the constraints, we incorporated a priori information of positivity and boundary condition (the reconstruction outside the initial target should be zero) in the reconstruction. The algorithm may provide a solution constrained by the above $a$ priori information. This is very important in the case with a limited solid angle like the case of laser fusion because the tomographic information of object can be increased due to incorporating a priori information. 


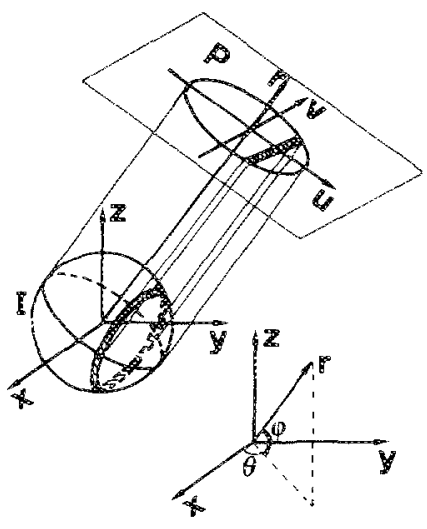

FIG. 1. Geometry of the coordinate system.

\section{RECOHSTRUCTION CAPABILITY}

The most important application of $\mathrm{CT}$ technique to laser fusion experiments is to estimate the size, shape, and position of the compressed core correctly. The reconstruction capability of this CT technique was checked by the computer simulations. Figure 2(a) shows the layergrams of the three-dimensional object used in the simulation. It consists of $49 \times 49 \times 49$ pixels. As shown in Fig. $2(a)$, the object contains two spheres of relative intensities 1,3 , and relative radii 24,9 , respectively. The central intense sphere has been deformed and shifted by 3 pixels from the center along for each $x, y$, and $z$ axis, respectively, on purpose. The imaging geometry is the same as that depicted (see Fig. 4). Figure 2(b) shows the reconstruction result after the 10 th iteration from five pinhole camera images (PHC 1-5 in Fig. 4). A good estimation of the original object has been obtained as shown in Fig. 2(b). We also checked the effect of noise contained in projections. We added a Poisson noise to each pinhole image. Figure 2(c) shows the result for signal-to-noise ratio $(S / N)$ of 10 . Ht can be seen that even the projections contain the noise, it is possible to estimate the size, shape, and position of the central core of the object. For the comparison, we also carried out the same simulations for the arrangement of three ( $\mathrm{PHC} \mathrm{1-3)}$ and four (PHC 1-4) pinhole images.

In order to evaluate the accuracy of the reconstruction, the averaged intensity error $\Delta I / I$ (root mean square, rms) and the averaged discrepancy of the size of center core

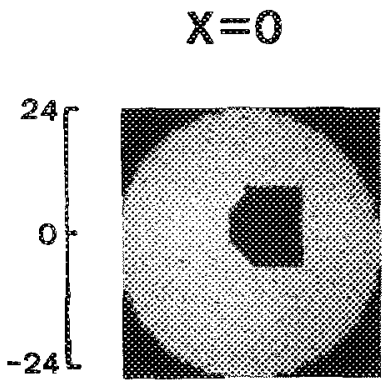

$$
x=5
$$
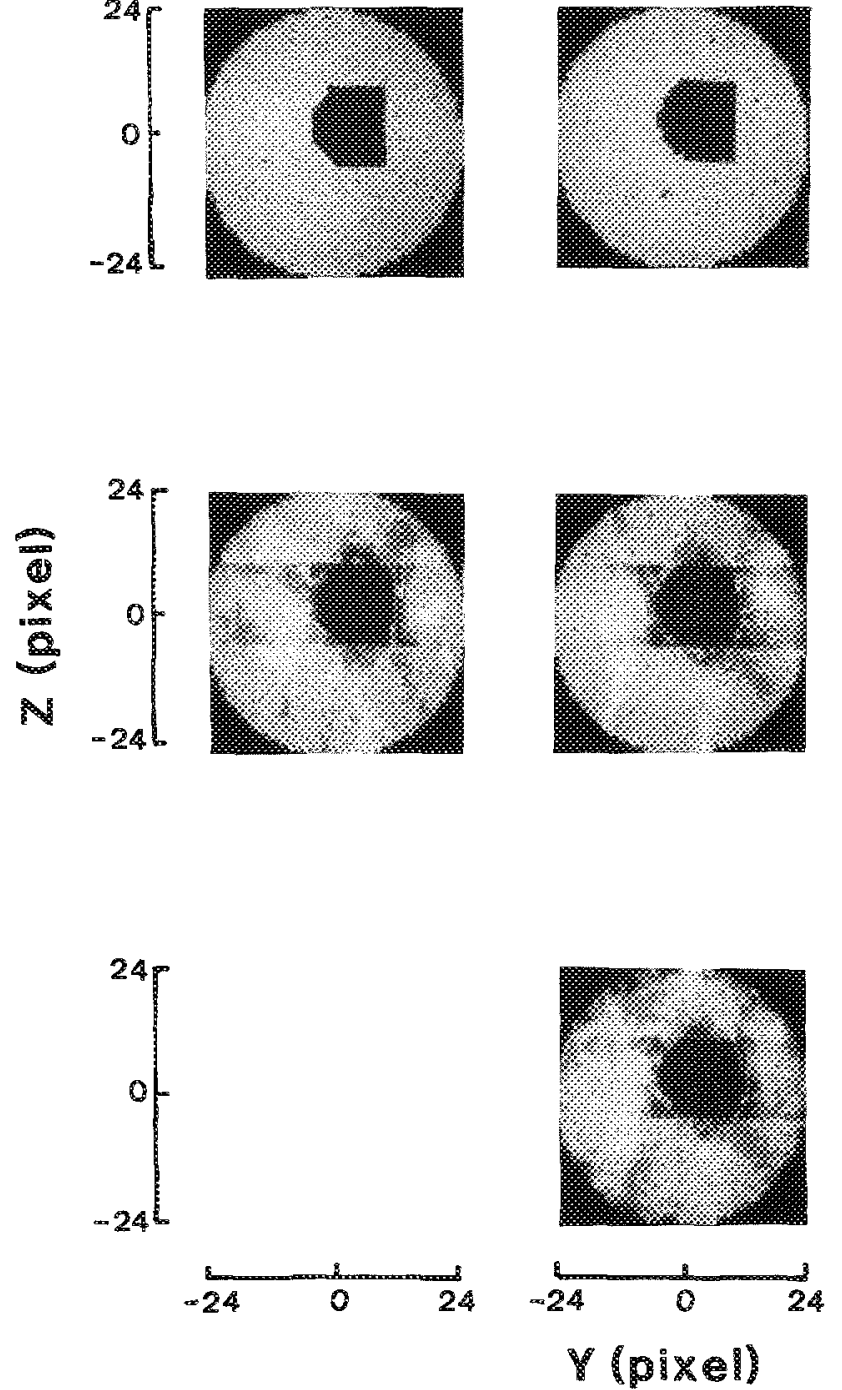

(b)
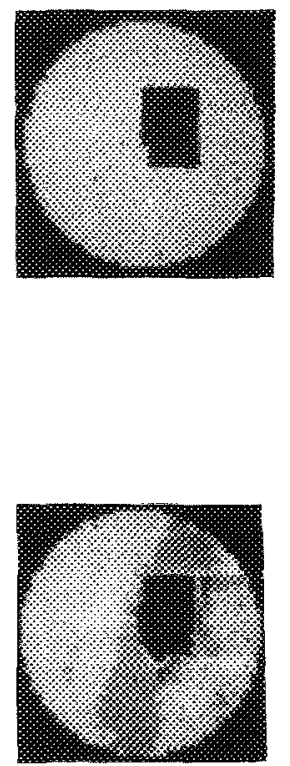

(a)
FIG. 2, (a) Test object, (b) reconstruction of the test object obtained from five pinhole images without noise, (c) reconstruction of the test object obtained from five pinhole images with noise $(S / N=10)$. 


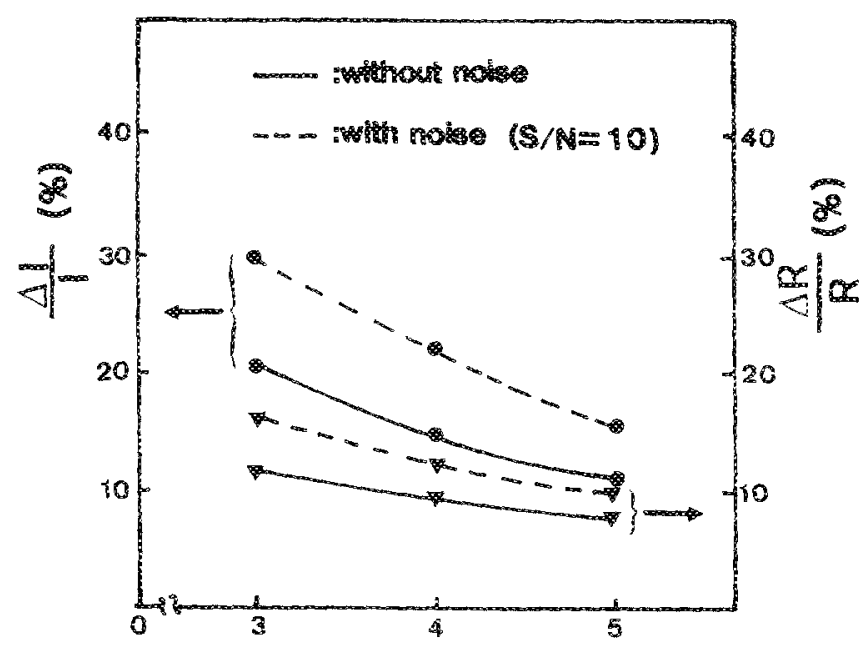

Number of viewing directions

FIG. 3. The intensity error $(\Delta I / T)$ and the core size discrepancy $(\Delta R / R)$ between the reconstructed image and original test object are improved with increasing the number of viewing directions.

$\Delta R / R$ between the original object and reconstructed image were used as measures. The discrepancies $\Delta I / I$ and $\Delta R / R$ are shown in Fig. 3. The dashed lines and solid lines show the accuracy of reconstruction with $(S / N=10)$ and without noise, respectively. As seer in this figure, the accuracy of the reconstruction is improved with the solid angle of the system. When we use five pinhole cameras, the discrepancies $\Delta I / I$ and $\Delta R / R$, were estimated to be $\sim 15 \%$ and $\sim 9 \%$ even for the noisy projection data $(S / N=10)$.

\section{B. EXPERIMENT}

At the demonstrations, we have applied this technique to reconstruct the three-dimensional image of imploded targets. The experiments were performed at the frequency doubled $(0.53 \mu \mathrm{m}) 12$ beam Nd: glass laser facillty, GEKKO XII ${ }^{8}$ at Osaka University. The target and laser data are presented in Table I for two representarive exper-

TABLE 1. Experimental conditions and results.

\begin{tabular}{|c|c|c|}
\hline & Shot A & Shot $\mathrm{B}$ \\
\hline $\begin{array}{l}\text { Target } \\
\text { diameter ( } \mathrm{mm} \text { ) } \\
\text { shell thickness ( } \mu \mathrm{m}) \\
\text { initial areal density } \\
\left(\mathrm{mg} / \mathrm{cm}^{2}\right)\end{array}$ & $\begin{array}{c}\text { CD shell target } \\
820 \\
5.35( \pm 1 \%) \\
5.35\end{array}$ & $\begin{array}{c}\text { Cr foam cryogenic target } \\
1000 \\
30( \pm 16 \%) \\
6\end{array}$ \\
\hline $\begin{array}{l}\text { Laser } \\
\text { cotal energy }(\mathrm{kJ}) \\
\text { wave length }(\mathrm{km}) \\
\text { pulse width (ns) }\end{array}$ & $\begin{array}{c}10 \\
0.53 \\
1.0\end{array}$ & $\begin{array}{c}10 \\
0.53 \\
1.0\end{array}$ \\
\hline $\begin{array}{l}\text { Results } \\
\text { neutron yield } \\
\text { (by Ag counter) } \\
\text { pR (mg/cm }{ }^{2} \text { ) } \\
\text { (by } 2 \text { nd reactions) }\end{array}$ & $8.59 \times 10^{9}$ & $3.12 \times 10^{8}$ \\
\hline
\end{tabular}

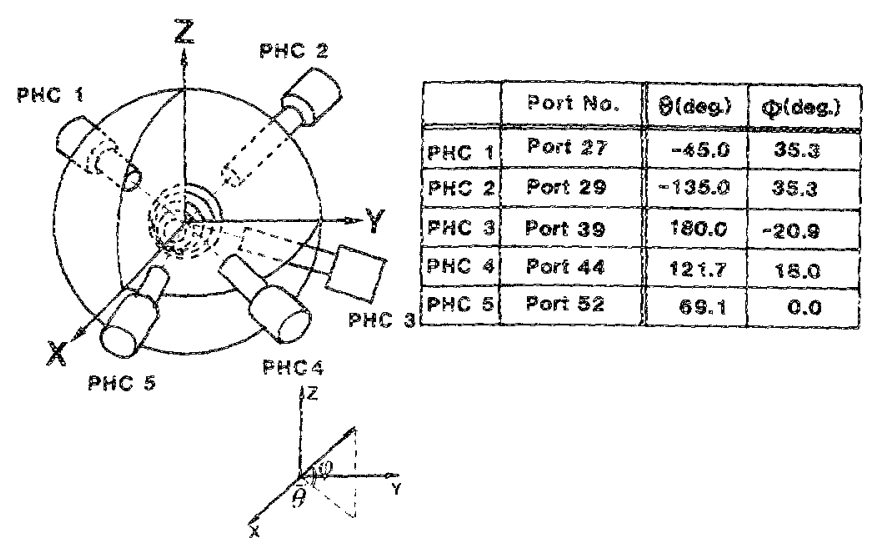

FIO. 4. Experimental setup for five viewing directions.

iments (designated shots $A$ and $B$ ), characterizing a uniform target (A) and a nonuniform target (B). The uniformities $(\Delta t / t: \Delta t=t-\bar{t})$ of sheil thickness $(t)$ for shots $A$ and $B$ were about $\sim 1 \%$ and $\sim 16 \%$, respectively. The experimental setup is shown in Fig. 4. Five pinhole cameras viewed the target at diferent observation directions. The distance between the pintoles and the target was 10 $\mathrm{cm}$ with the camera magnifications of $\times 6$. The aperture of each pinhole camera was $15 \mu \mathrm{m}$ in diameter drilled in a 40- 2 m-thick tantalum. A 10- $\mu$ m-thick Al filter and a 20 um-thick Be filter were placed in front of the film plane. X rays greater than $1.5 \mathrm{keV}$ were recorded on the $\mathrm{x}$-ray film (Fuji MI-FX). Each pinhole image was digitized into a $49 \times 49$ array using a $2-D$ densitometer. The digitized pinhole images were corverted to intensity (photon $/ \mathrm{cm}^{2}$ ) using the H-D curve of Fuji MI-FX for $1.5 \mathrm{keV}$.?

The total areal densities of plasma $\rho R$, which may ato fect the transmission of $x$-rays, were measured to be 1.8 $\mathrm{mg} / \mathrm{cm}^{2}$ for shot $\mathrm{A}$ and $2.1 \mathrm{mg} / \mathrm{cm}^{2}$ for shot 3 by the secondary reaction method. ${ }^{10}$ The transmittance of $1.5 \mathrm{keV}$ $x$-rays is estimated to be nearly $100 \%$ for these low areal densities using the free-iree absorption formulas, ${ }^{11}$ with the simulated temperature of $1.0 \mathrm{keV}$.

In the process of recorstruction from the five pinhole images, a priori informations of positivity and boundary condition (the reconstruction outside the initial target should be zero) have beer: imposed. To reduce the effect of noise contained in the pinhole images, a smoothing step was inserted at each iteration. The smoothing kemel consists of 7 pixels (one central and 6 nearest neighbors) with the weighted value of the central pixel five times larger than that of its neighbors. The reconstructions were obtained after 10 iterations.

Figures $5(a)$ and $5(b)$ show iso-intensity (a half of maximum intensity) surfaces of the reconstructed compressed core of shots $A$ and $B$, respectively. The pixel size was $20 \mu \mathrm{m}$, and the spatial resolution of the reconstruction of compressed core was calculated to be $32 \mu \mathrm{m}$ as a point response (FWHM), taking into account of the effect of noise contained in projections such as film grains and statistical noise $(S / N=10)$. 

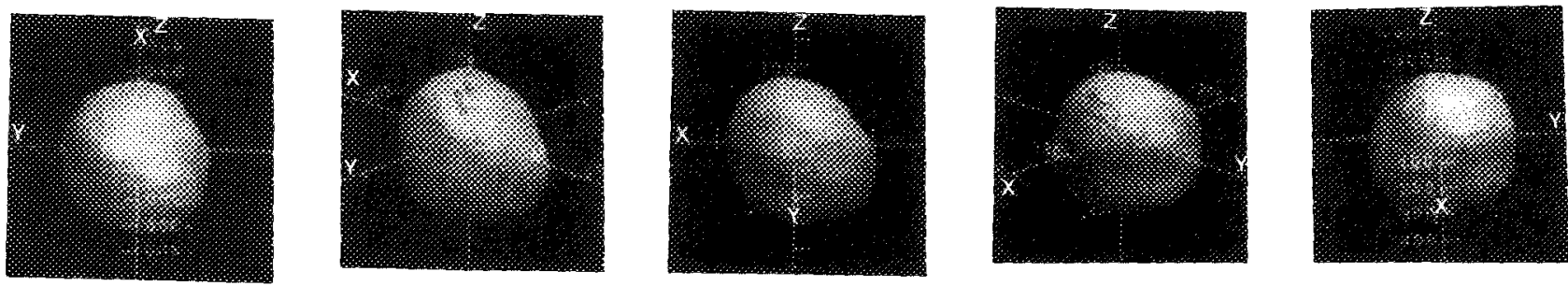

(a)
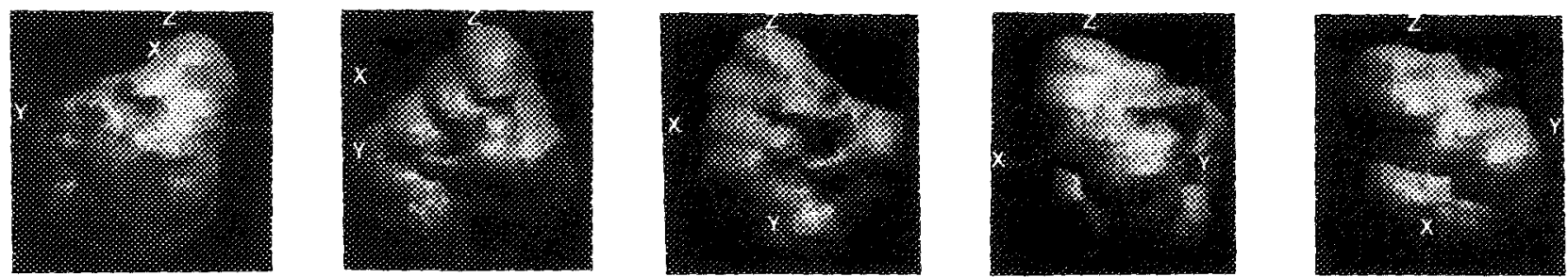

(b)

FIG. 5. The isointensity (the half of maximum intensity) surface of the 3-D images of compressed core. (a) uniform shell target (shot A), (b) nonuniform shell target (shot B).

\section{DISCUSSION}

The uniformity and size of the compressed core are estimated from a pinhole image which is a two-dimensional projection of the three-dimensional compressed core. This is impossible to evaluate the uniformity and core size correctly. From Fig. 5, we can now clearly recognize the shape and size of the compressed core. The averaged intensity FWHM of the compressed cores are estimated to be about 110 and $240 \mu \mathrm{m}$ for Figs. $S(a)$ and $5(b)$, respectively. In order to evaluate the uniformity of the compressed core, the radial distributions of the compressed cores (as shown in Fig. 5) were expressed in the expansion by the spherical harmonics. The mode expansions of the compressed core radius distributions are shown in Fig. 6. The rms values $\sigma$ of the radius distributions of shots $A$ and $B$ were estimated to be $\sim 17 \%$ and $\sim 46 \%$, respectively. Because the accuracy $\Delta R / R$ of the $C T$ reconstruction is about $9 \%$, one can also easily recognize that the spherical symmetry has been much better for shot $\mathrm{A}$ than for shot $\mathrm{B}$. Especially, it should be noted that the $\sigma$ of the low mode is larger for shot $\mathrm{B}$ than for shot $\mathrm{A}$.

The laser energy balances for both shots $A$ and $B$ were $\pm 3 \%$. The considerable difierence which may affect the uniformity of the compressed core is the uniformity of initial target shell thickness. The shell thickness distribution of shot $B$ is shown in Fig. 7(b) and a layergram of the three-dimensional reconstruction of shot $B$ at the center is shown in Fig. $7(a)$ for comparison. The observing direction is the same for both Figs. $7(a)$ and $7(b)$. It can be seen that the compressed core is shifted towards the side of thicker part of the shell. This observation tells us that the

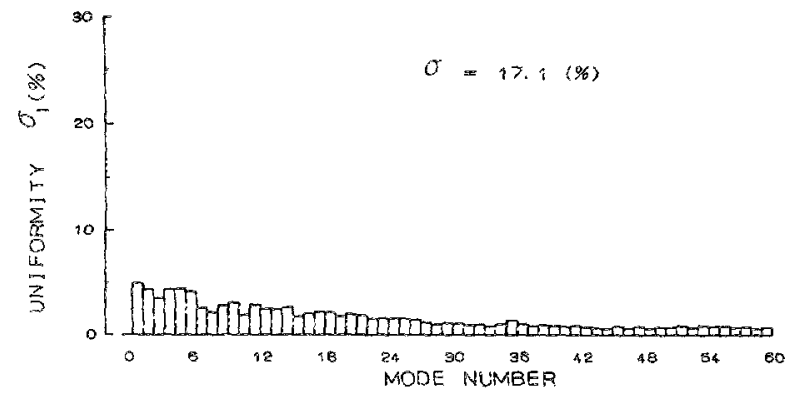

(a)

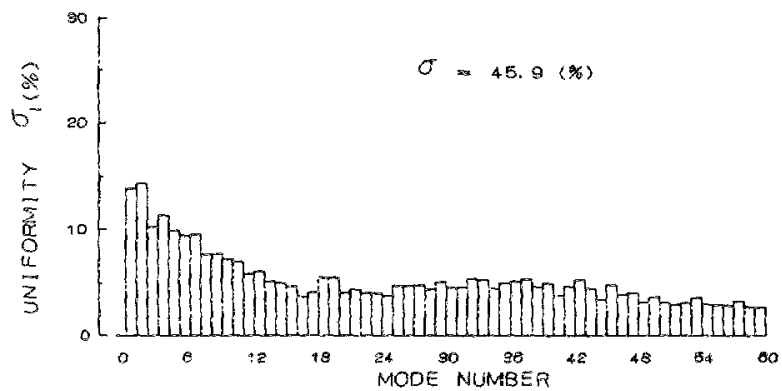

(b)

FIG. 6. Mode expansions of the compressed core radius distribution (as shown in Fig. 5) into the spherical harmonics. (a) uniform shell target (shot $A$ ), (b) nonuniform shell target (shot B). 




(a)

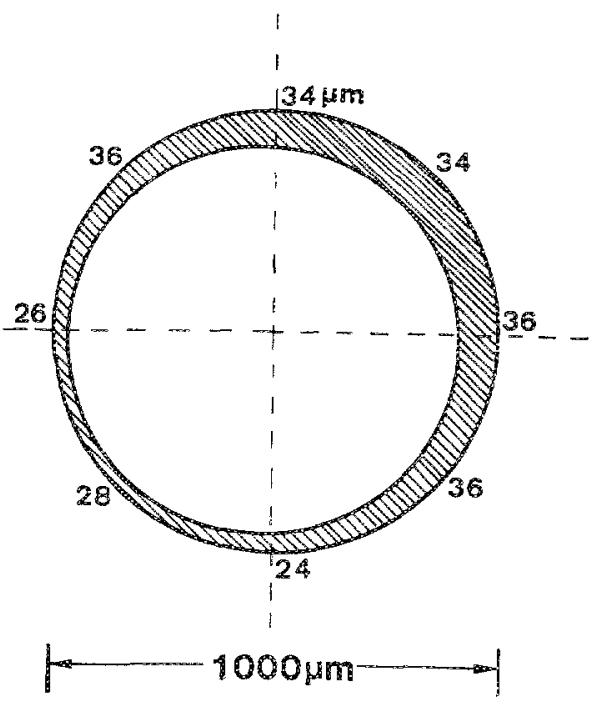

(b)
FIG. 7. (a) Layergram of the 3-D reconsiructed image (shot B) at the center and (b) distribution of initial target shell thickness. uniformity of the compressed core strongly correlates with the uniformity of shell thickness.

The effects of the nonuniformity of target shell thickness on the implosion uniformity have been simulated by using a two-dimensional Lagrangian hydrodynamic code HISHO-20, ${ }^{12}$ which used an average-atom ionizationequilbrium mode ${ }^{13}$ to describe the atomic physics and radiation transport. The target used in this simulation was the same as the shot $B$ as shown in Table $I$. We incorporated a spherical harmonic (mode 1) into the target shell thickness profle as shown in Fig. $8(a)$. This harmonic simuiates in a simple manner the $\pm 16 \%(\Delta t= \pm 5 \mu \mathrm{m})$ nonuniformity of the real target shell thickness. When this target is irradiated by a $10 \mathrm{~kJ}$ green laser light of $1.0 \mathrm{~ns}$ (Gaussian, FWHM), the maximum compression is found at $t=1.0 \mathrm{~ns}$ after the laser peak. The snap shots of the implosion dynamics at $t=0.6 \mathrm{~ns}$ and $t=1.0 \mathrm{~ns}$ (maxi= mum compression) after the laser peak are shown in Figs. $8(b)$ and $8(c)$, respectively. It is seen that the nonuniformity of implosion grows with the convergence of the shell. At the maximum compression, the predicted compressed core is similar to the observed one, which is shifted towards the side of thicker target shell thickness. The compressed cores (at maximum compression) simulated with different nonuniformities of the shell thickness of a cryogenic foam target are shown in Fig. 9 . It is clear that as the nonuniformity of target shell thickness is increased, the deforma tion of compressed core is increased. The ratio of the fue? density $\rho$ for each case of nonuniform target to the fuel density $\rho$ of uniform target are shown in Fig. 10. It can be seen that the nonuniformity of shell thickness causes a significant reduction of fuel density. In order to achieve a high fuel density, the uniformity of shell thickness should be improved to be better than about $3 \%$.

The good agreement between the simulation results and the experimental results strongly suggests that unifor-

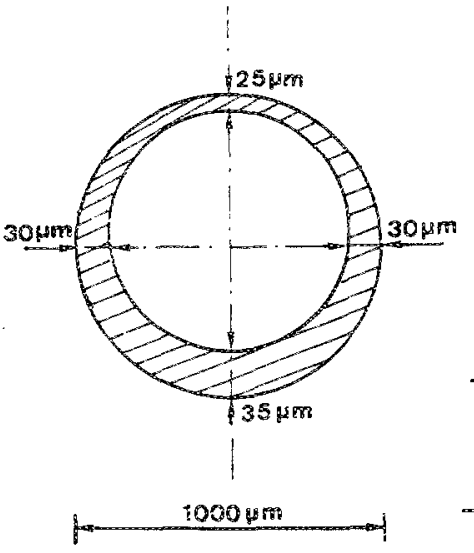

(a)

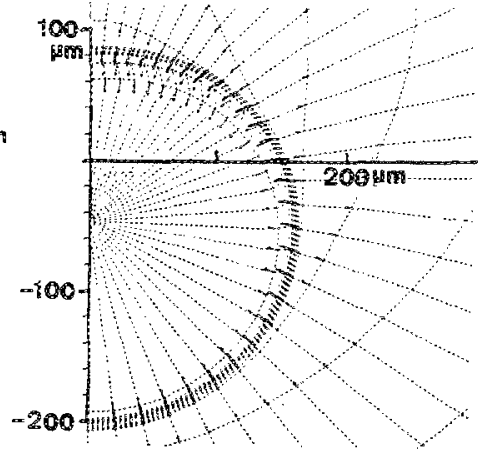

(b)



(c)
FIG. 8. A two-dimensional hydrodynamic simulation. (a) Distribution of initial target shell thickness (mode 1) (b) and the snap shots at $0.6 \mathrm{~ns}$, (c) $1.0 \mathrm{~ns}$ (maximum compression) after laser peak. 


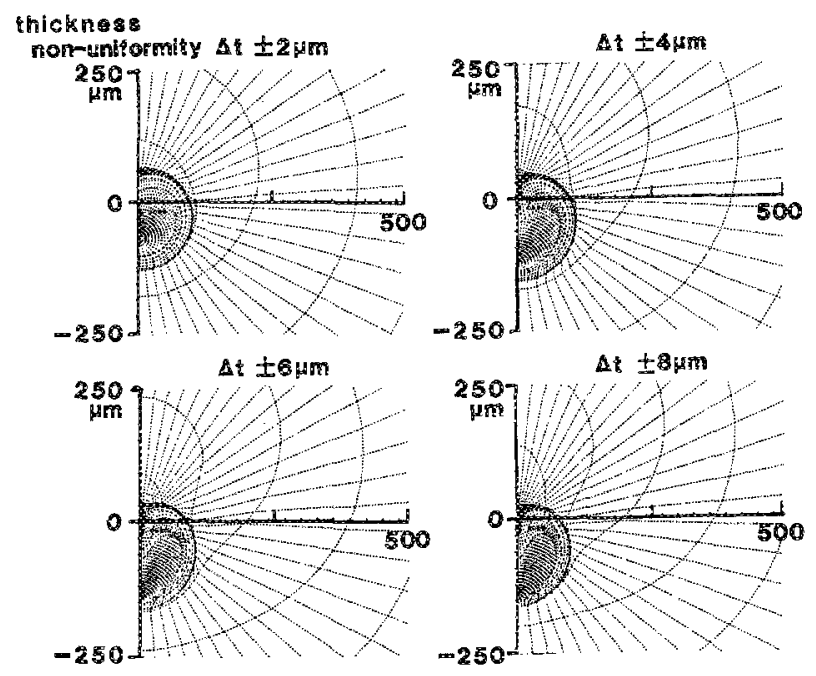

FIG. 9. Calculated nonuniformity of compressed core for the different thickness nonuniformities $\Delta t$ of the shell.

mity of initial target shell thickness is crucial in achieving good implosion symmetry.

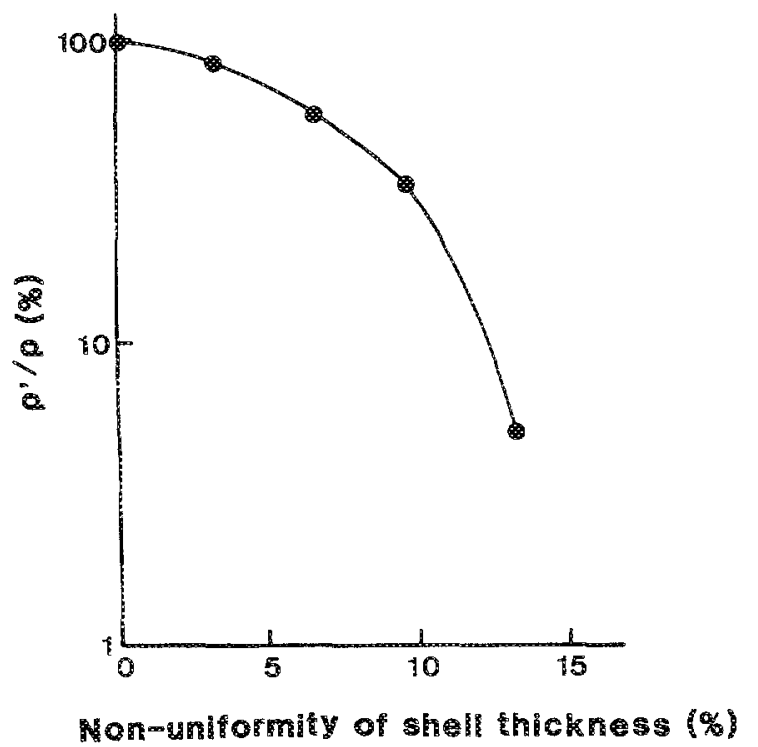

FIG. 10. Reduction of compressed fuel density due to the nonmiformity of shell thickness.

\section{CONCLUSION}

A computed tomographic technique using five pinhole cameras has been applied to laser fusion experiments to reconstruct a three-dimensional $x$-ray image of imploded targets. Direct evidences of a deviation from the spherically symmetric core due to the nonuniformity of target shell thickness have been observed. The observation agrees well with that calculated by two-dimensional hydrodynamic simulations incorporating the spherical harmonic into the target sheil thickness profile. Future efforts using more viewing directions than five will improve the spatial resolution of the reconstruction to $10 \mu \mathrm{m}$ to allow imaging of targets that achieve high radial convergence. This technique will also be applied to obtain a time-resolved threedimensional image of the compressed core by using $x$-ray multiframing cameras.

\section{ACKNOWLEDGMENTS}

We wish to thank Y. Fukuda, K. Shima, K. Sawai, M. $\mathrm{Kawaguchi}$, and $\mathbb{R}$. Hashimoto for their helpful discussions on computer calculation. We also thank the GEKKO XII laser operation group, diagnostic group and target fabrication group.

'N. M. Ceglio and L. W. Coleman, Phys. Rev. Lett. 39, 20 (1977).

${ }^{2}$ Y. W. Chen, N. Myanaga, M. Yamanaḱsa, H. Azechi, K. Nishihara, Y. Kato, T. Yamanaka, and S. Nakai (unpublished).

${ }^{3}$ R. A. Brooks and G. Chiro, Phys. Med. Biol. 21, 659 (1976).

${ }^{4} \mathrm{H}$. Nishimura, H. Niki, N. Miyamaga, K. Okada, H. Azechi, T. Yabe, R. Tsuji, S. Ido, M. Yamanaka, T. Mochizuki, K. Nishiara, T. Yamanaka, and C. Yamanaka, Rev. Sci. Instrum. 56, 1128 (1985).

${ }^{5}$ Y.-W. Chen, M. Yamanaka, N. Miyanaga, T. Yamanaka, S. Nakai, C. Yamanaka, and S. Tamura, Opt. Commun. 71, 249 (1989).

${ }^{\circ} \mathrm{G}$. T. Herman, A. Lent, and S. W. Rowiand, J. Theor. Biol. 42, I (1973).

${ }^{7}$ A. C. Kak, Proc. IEEE 67, 1245 (1979).

${ }^{8}$ Y. Kato, Y. Kanabe, T. Yamada, S. Sakabe, S. Urushibara, H. Azechi, and $N$. Miyanaga, Institute of Laser Engineering, Quarterly Progress Report, ILE-QPR-84-8, Osaka University (unpublished).

${ }^{9}$ N. Miyanaga, Ph.D. dissertation, Osaka University, 1987 (unpublished).

${ }^{10} \mathrm{H}$. Azechi, N. Miyanaga, R. O. \$tapf, K. Itoga, H. Nakaishi, M. Yamanaka, H. Shiraga, R. Tsuji, \$. Ido, K. Nishihara, Y. Izawa, T. Yamanaka, and C. Yamanaka, Appl. Phys. Lett. 49, 5551 (1986).

${ }^{11}$ Yr.B.Zel'dovich and Yu.P.Raizer, Physics and Shock Woves and HighTemperature Hydrodynamic Phenomena (Academic, New York, 1966).

${ }^{12} \mathrm{~K}$. Nishihara (private communication).

${ }^{13}$ M. Murakami and K. Nishihara, Jpn. J. Appl. Phys. 26, 1132 (1987). 